\title{
Magnetic Anisotropy in Functionalized Bipyridyl Cryptates
}

\author{
Elisabeth Kreidt, ${ }^{a}$ Caroline Bischof, ${ }^{b}$ Carlos Platas-Iglesias, ${ }^{* c}$ and \\ Michael Seitz ${ }^{*}$ \\ ${ }^{a}$ Institute of Inorganic Chemistry, University of Tübingen, Auf der \\ Morgenstelle 18, 72076 Tübingen, Germany. \\ ${ }^{b}$ Inorganic Chemistry I, Ruhr-University Bochum, 44780 Bochum, \\ Germany. \\ c Departamento de Química Fundamental, Universidade da Coruña, \\ Campus da Zapateira-Rúa da Fraga 10, 15008 A Coruña, Spain
}

Email: michael.seitz@uni-tuebingen.de, carlos.platas.iglesias@udc.es

\section{Supporting Information}

Table of Contents

Page

1. NMR Spectra

S2

2. HPLC traces of $\mathbf{5 - L n}$

S9

3. DFT Calculations

S10 


\section{NMR Spectra}

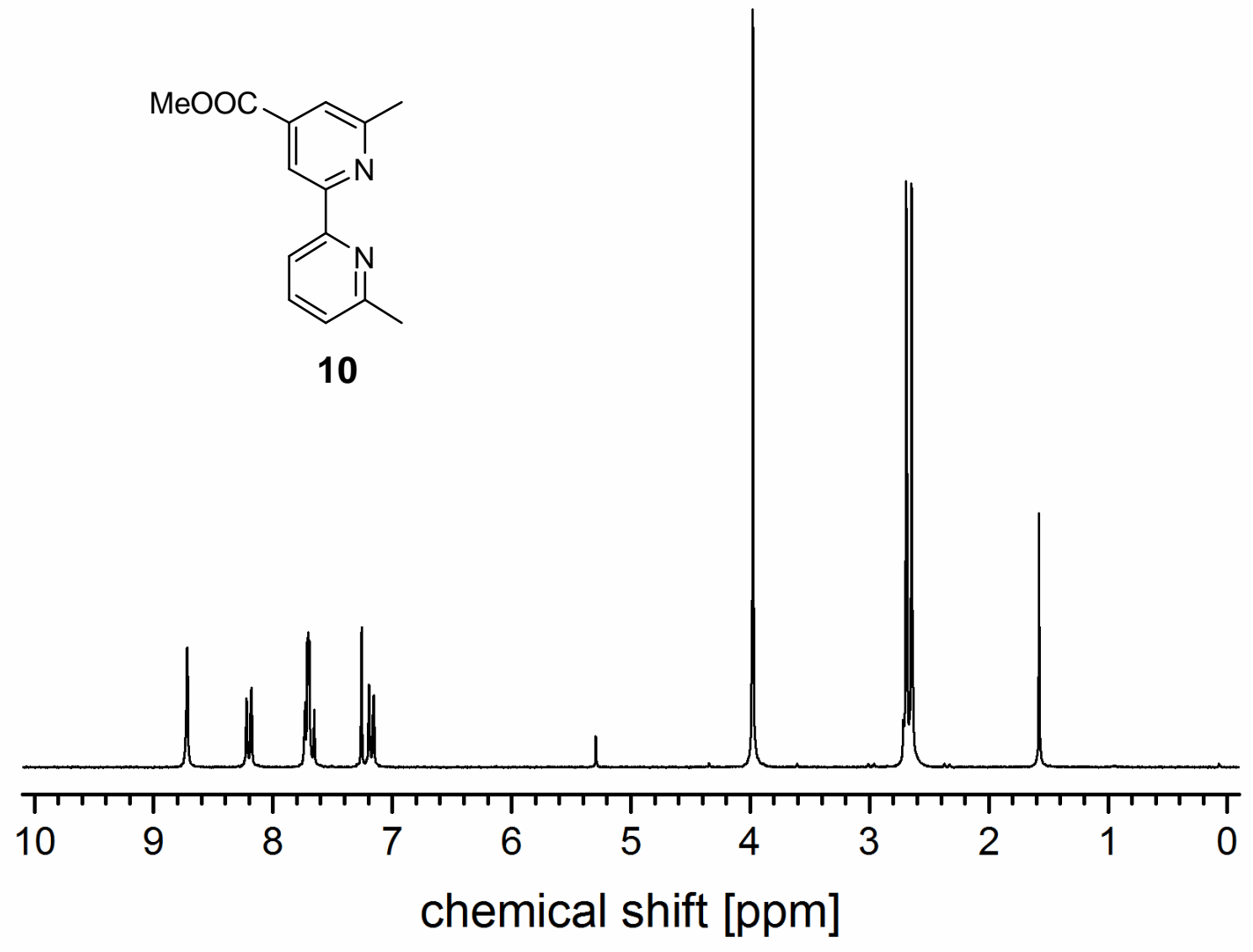

Figure S1. ${ }^{1} \mathrm{H}$ NMR $\left(200 \mathrm{MHz}, \mathrm{CDCl}_{3}\right)$ of 10.<smiles>CC(=O)c1cc(C)nc(-c2cccc(C)n2)c1</smiles>

11

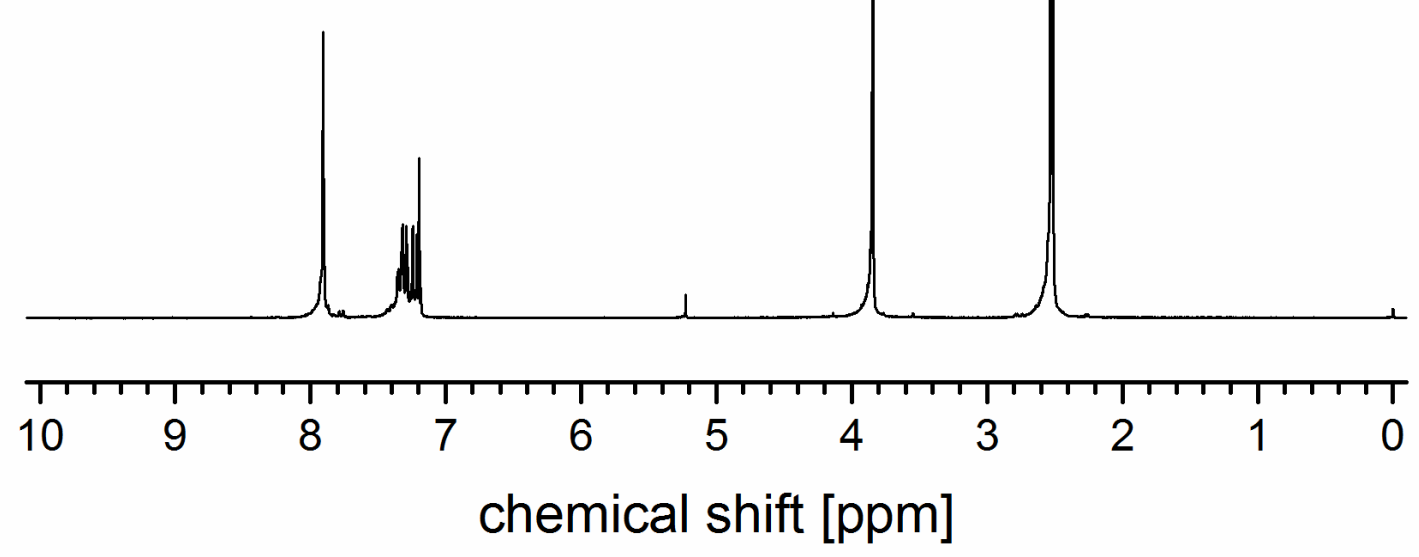

Figure S2. ${ }^{1} \mathrm{H}$ NMR $\left(250 \mathrm{MHz}, \mathrm{CDCl}_{3}\right)$ of 11. 


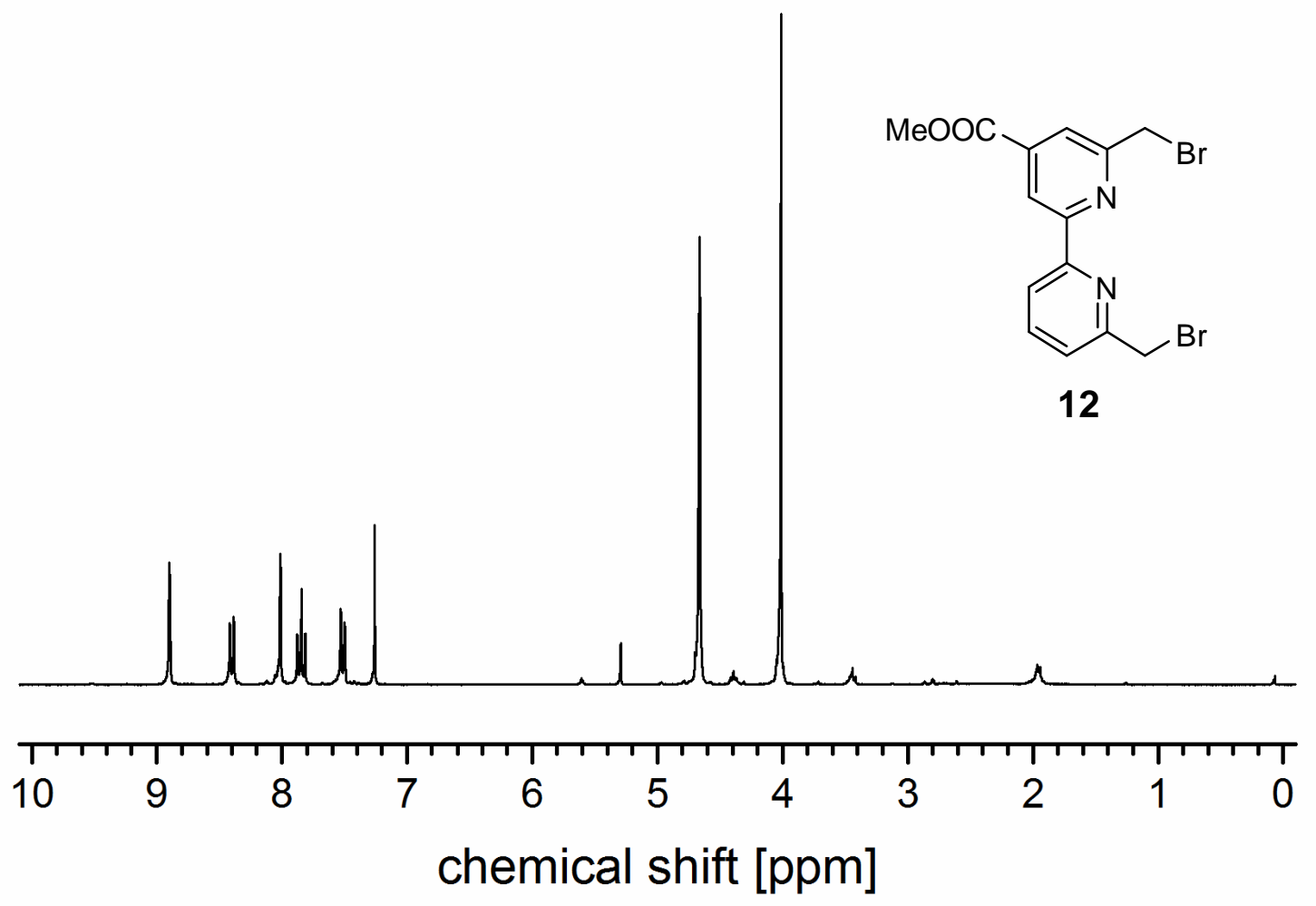

Figure S3. ${ }^{1} \mathrm{H}$ NMR $\left(200 \mathrm{MHz}, \mathrm{CDCl}_{3}\right)$ of 12.

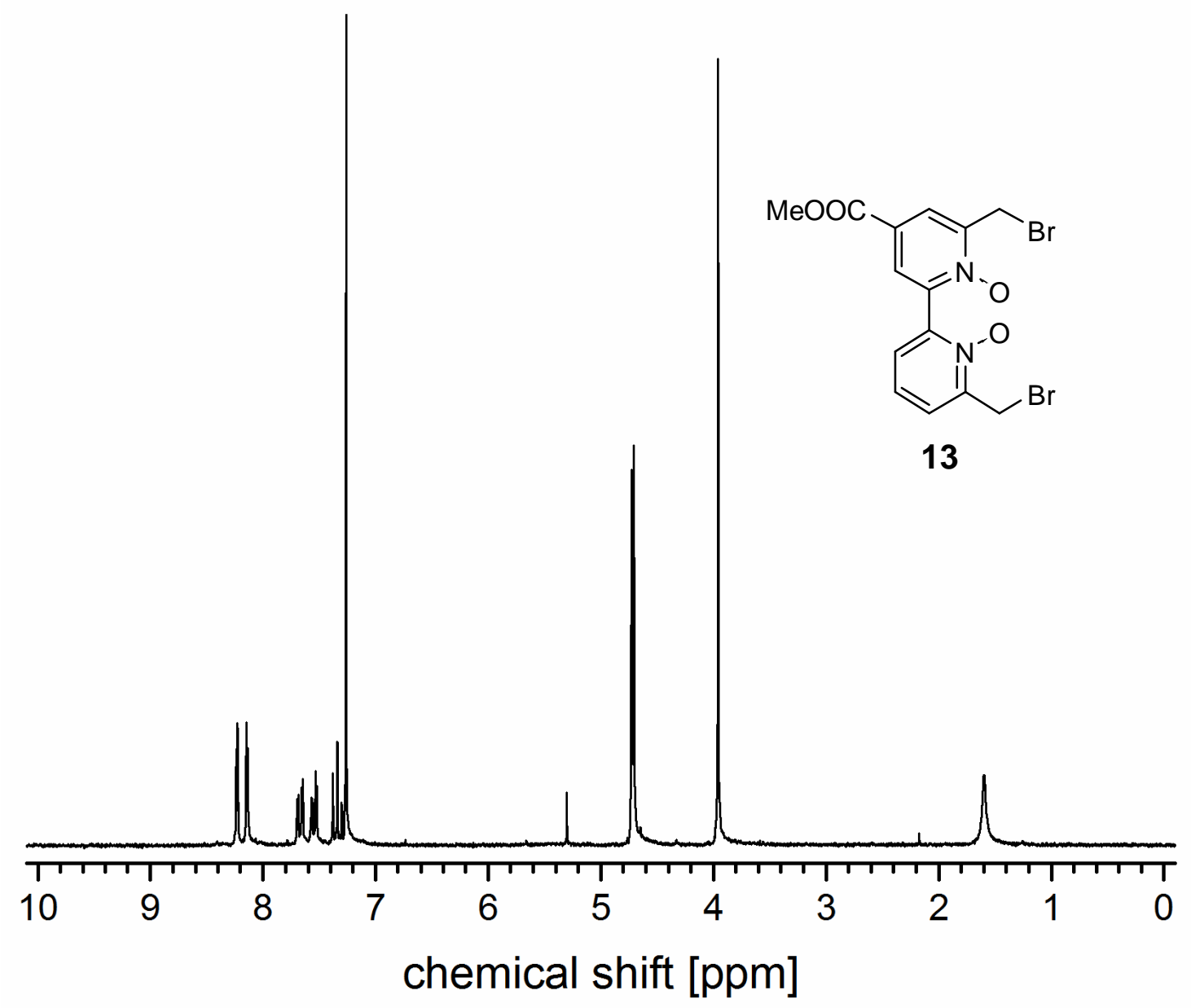

Figure S4. ${ }^{1} \mathrm{H} \mathrm{NMR}\left(200 \mathrm{MHz}, \mathrm{CDCl}_{3}\right)$ of 13. 


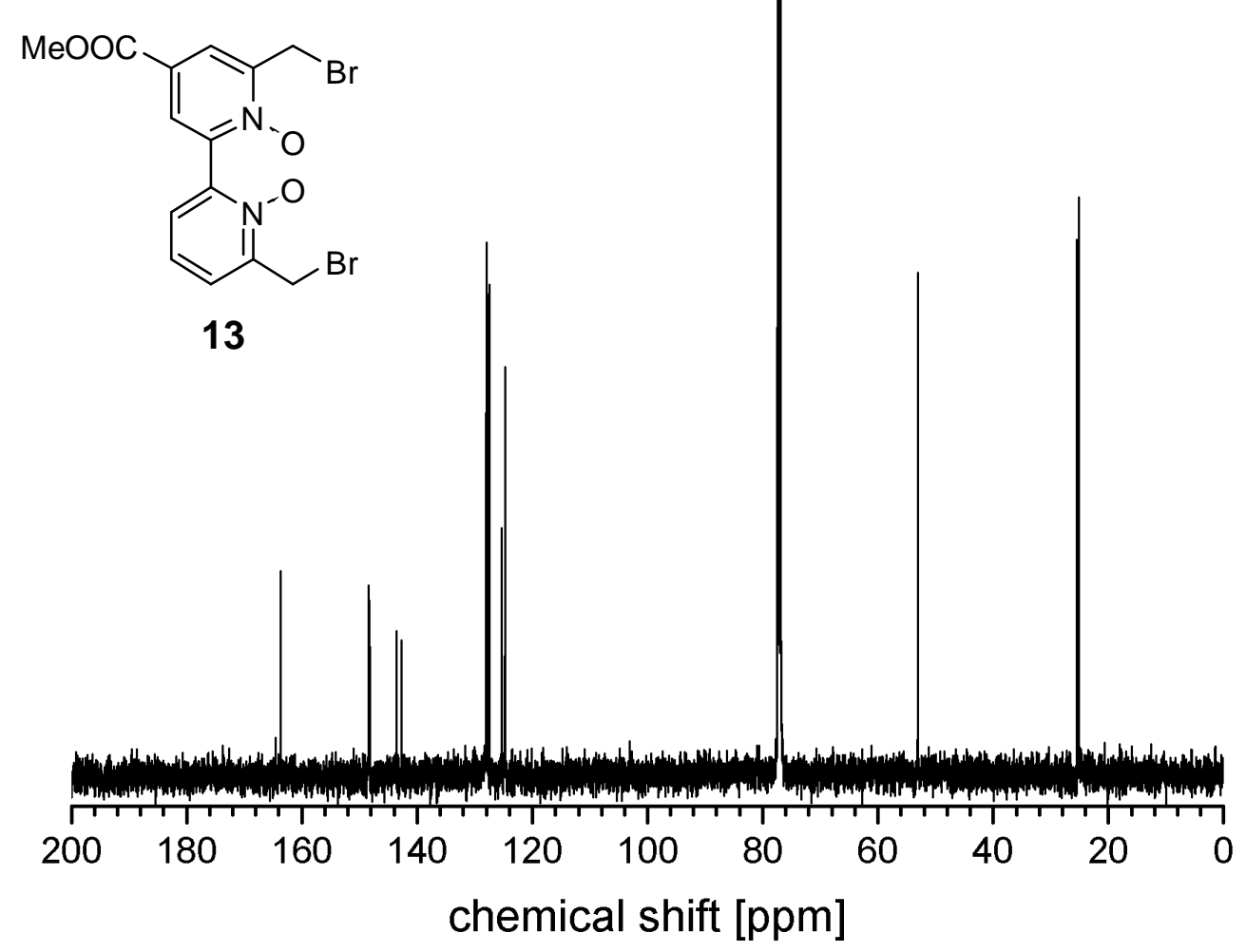

Figure S5. ${ }^{13} \mathrm{C}$ NMR (126 MHz, $\left.\mathrm{CDCl}_{3}\right)$ of 13.

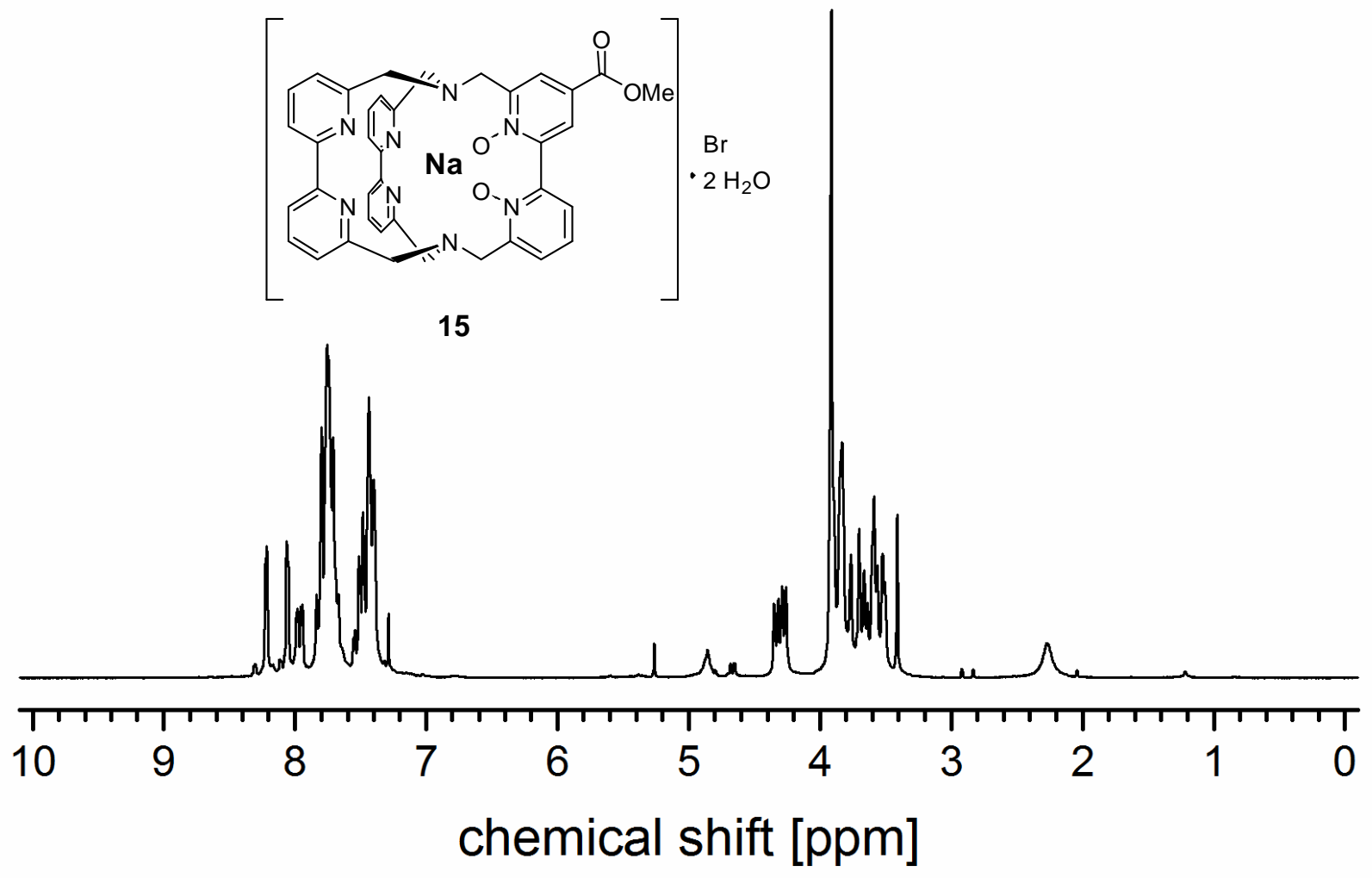

Figure S6. ${ }^{1} \mathrm{H} \mathrm{NMR}\left(200 \mathrm{MHz}, \mathrm{CDCl}_{3}\right)$ of 15. 


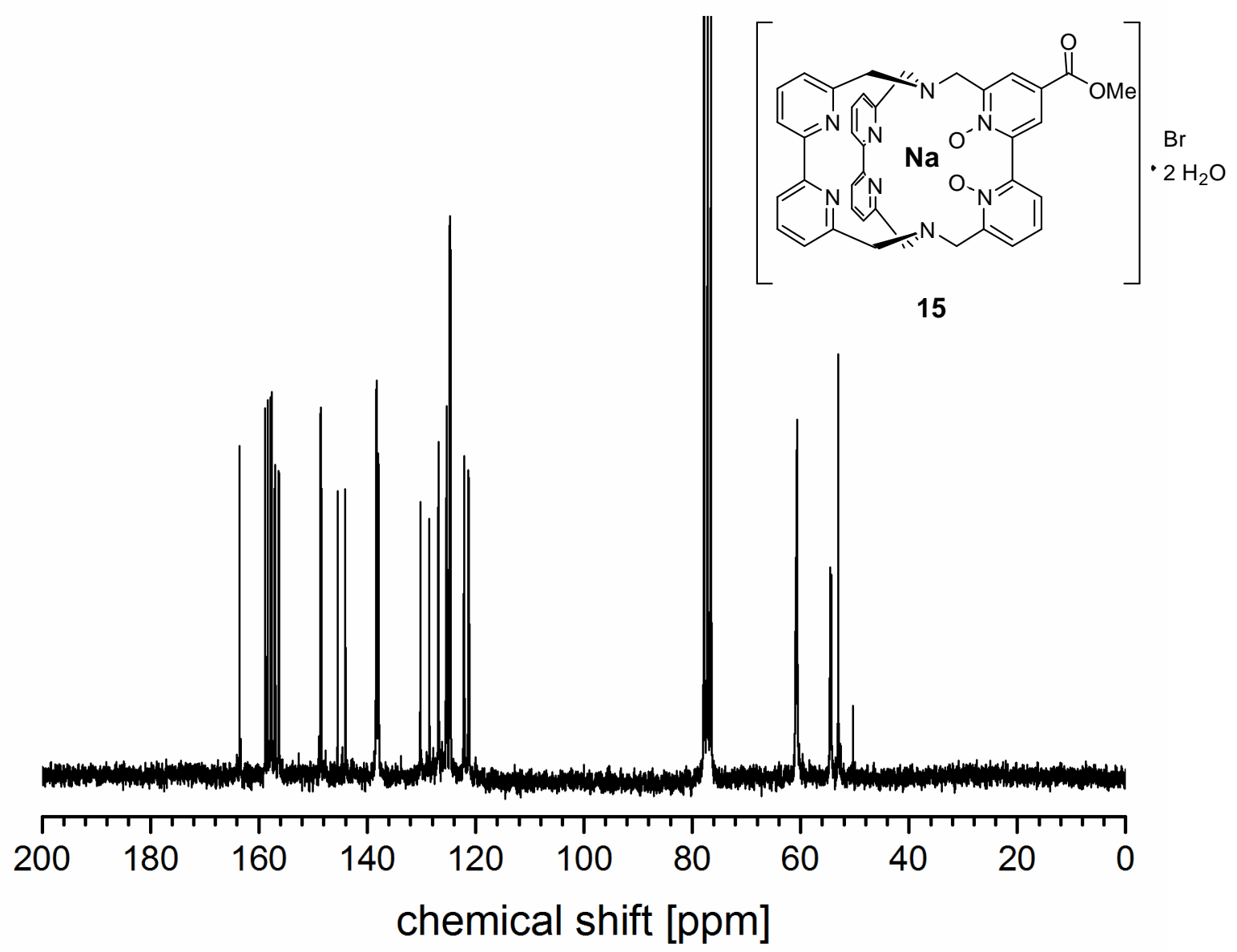

Figure S7. ${ }^{13} \mathrm{C}$ NMR $\left(50.3 \mathrm{MHz}, \mathrm{CDCl}_{3}\right)$ of $\mathbf{1 5}$.

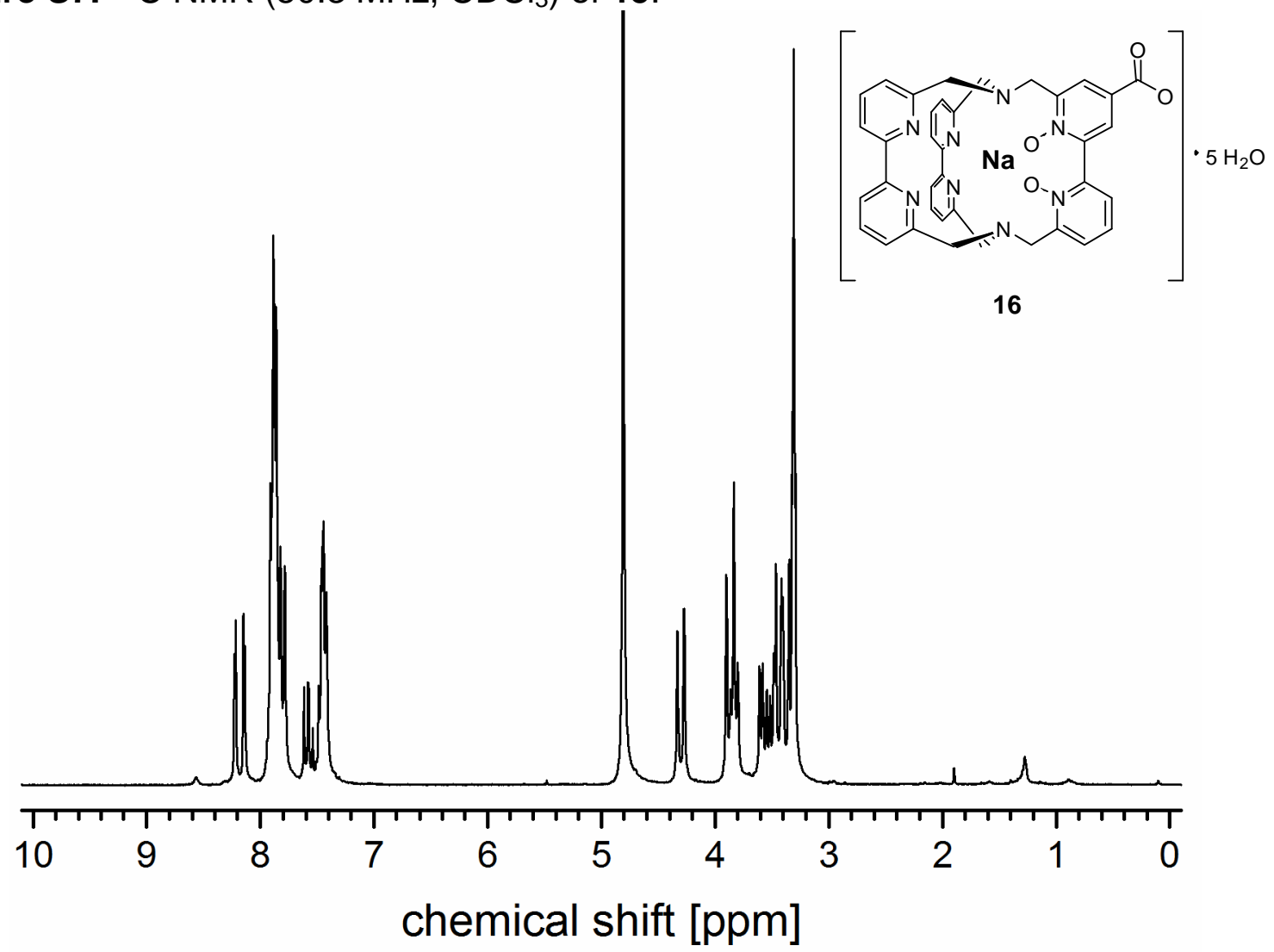

Figure S8. ${ }^{1} \mathrm{H}$ NMR $\left(200 \mathrm{MHz}, \mathrm{CD}_{3} \mathrm{OD}\right)$ of 16. 


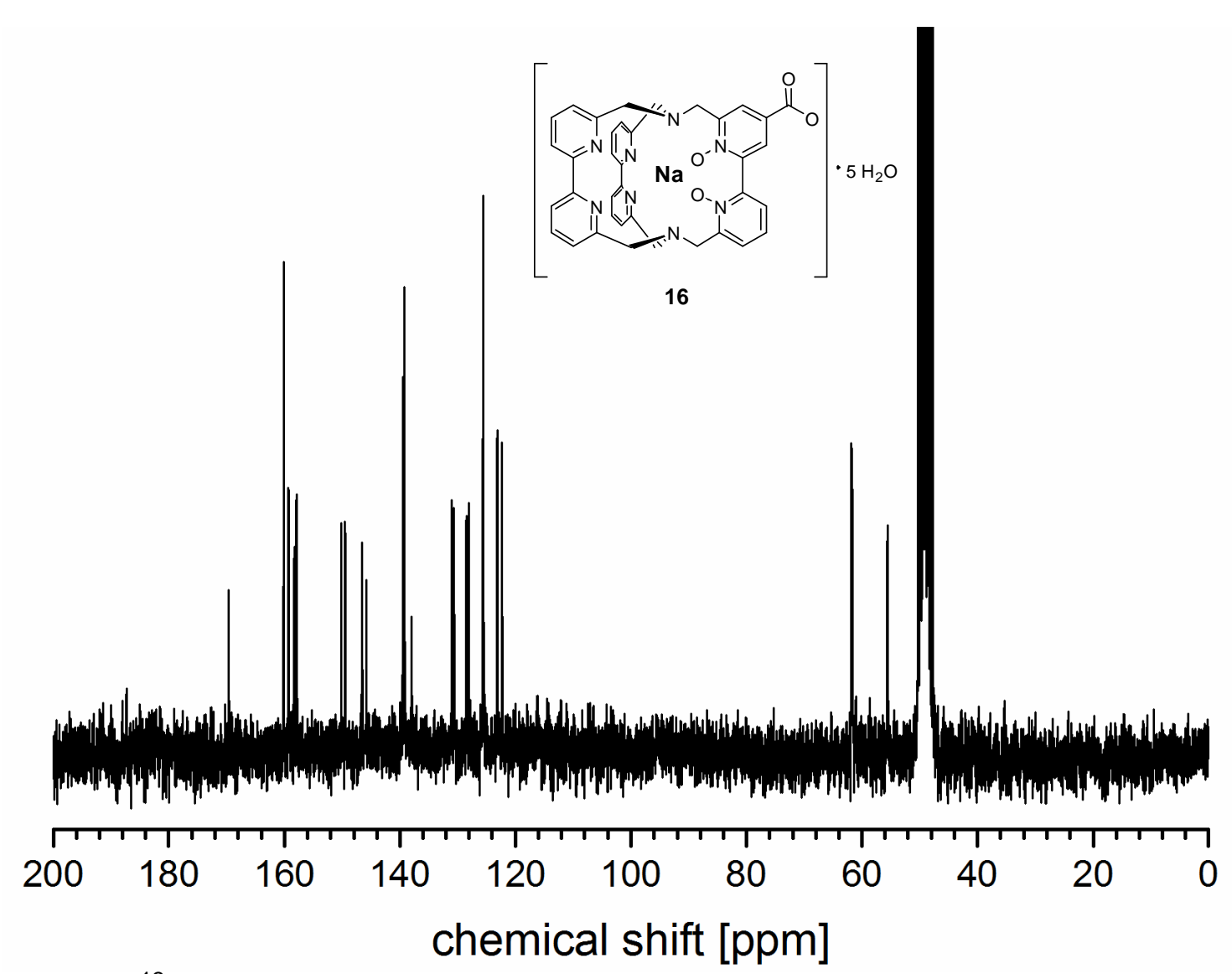

Figure S9. ${ }^{13} \mathrm{C} \mathrm{NMR}\left(50.3 \mathrm{MHz}, \mathrm{CD}_{3} \mathrm{OD}\right)$ of 16.

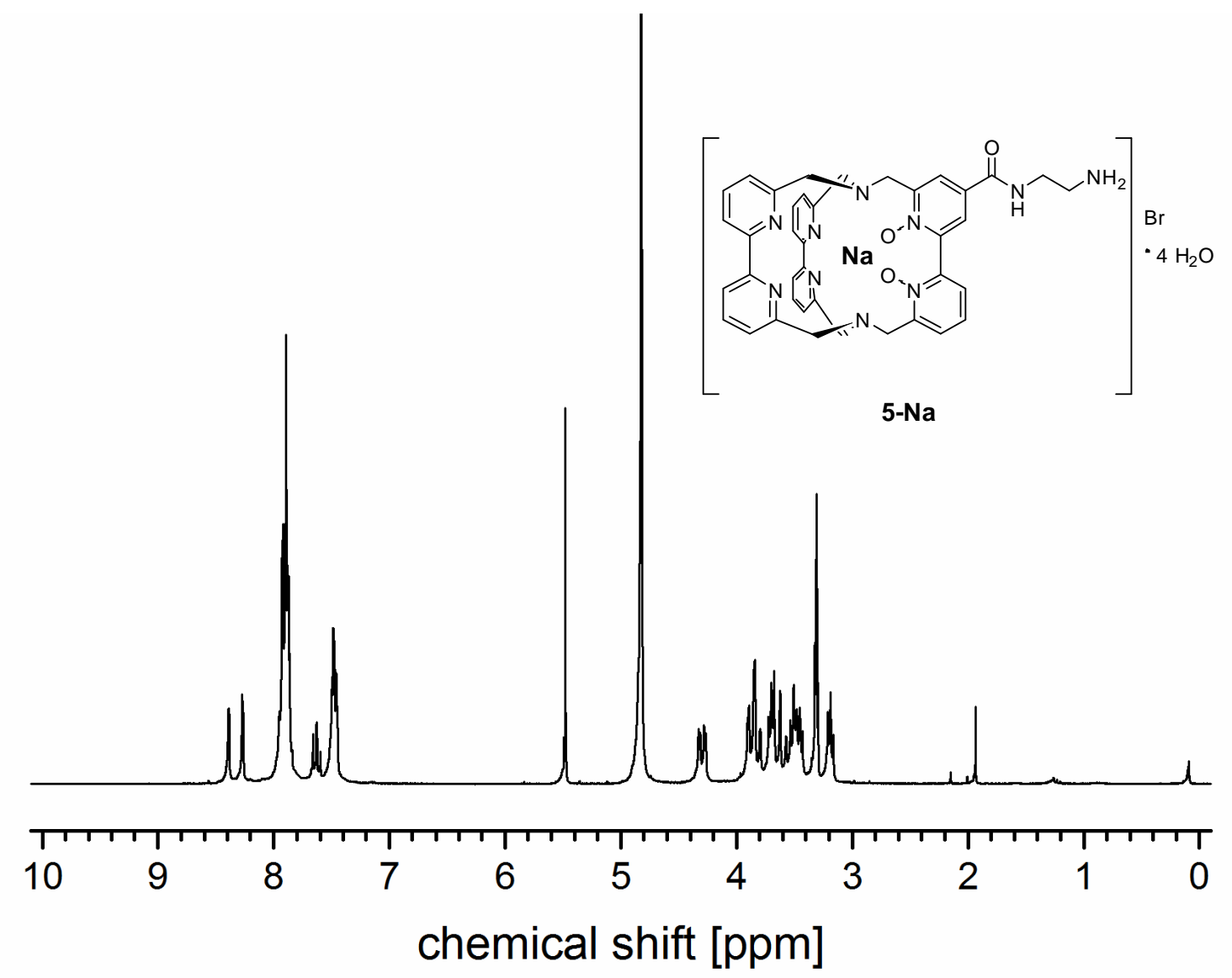

Figure S10. ${ }^{1} \mathrm{H} \mathrm{NMR}\left(250 \mathrm{MHz}, \mathrm{CD}_{3} \mathrm{OD}\right)$ of 5-Na. 


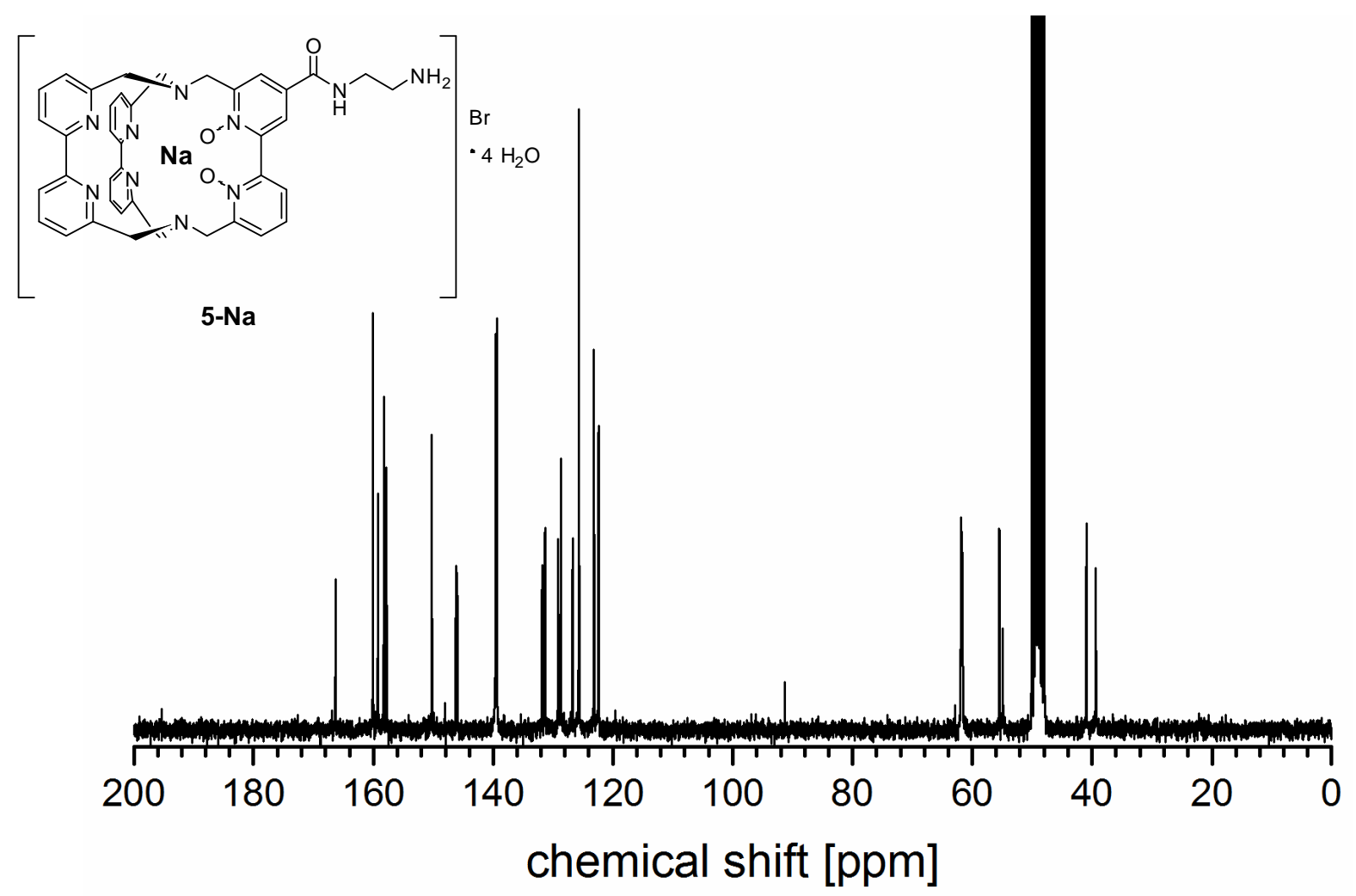

Figure S11. ${ }^{13} \mathrm{C}$ NMR $\left(62.9 \mathrm{MHz}, \mathrm{CD}_{3} \mathrm{OD}\right)$ of 5-Na.

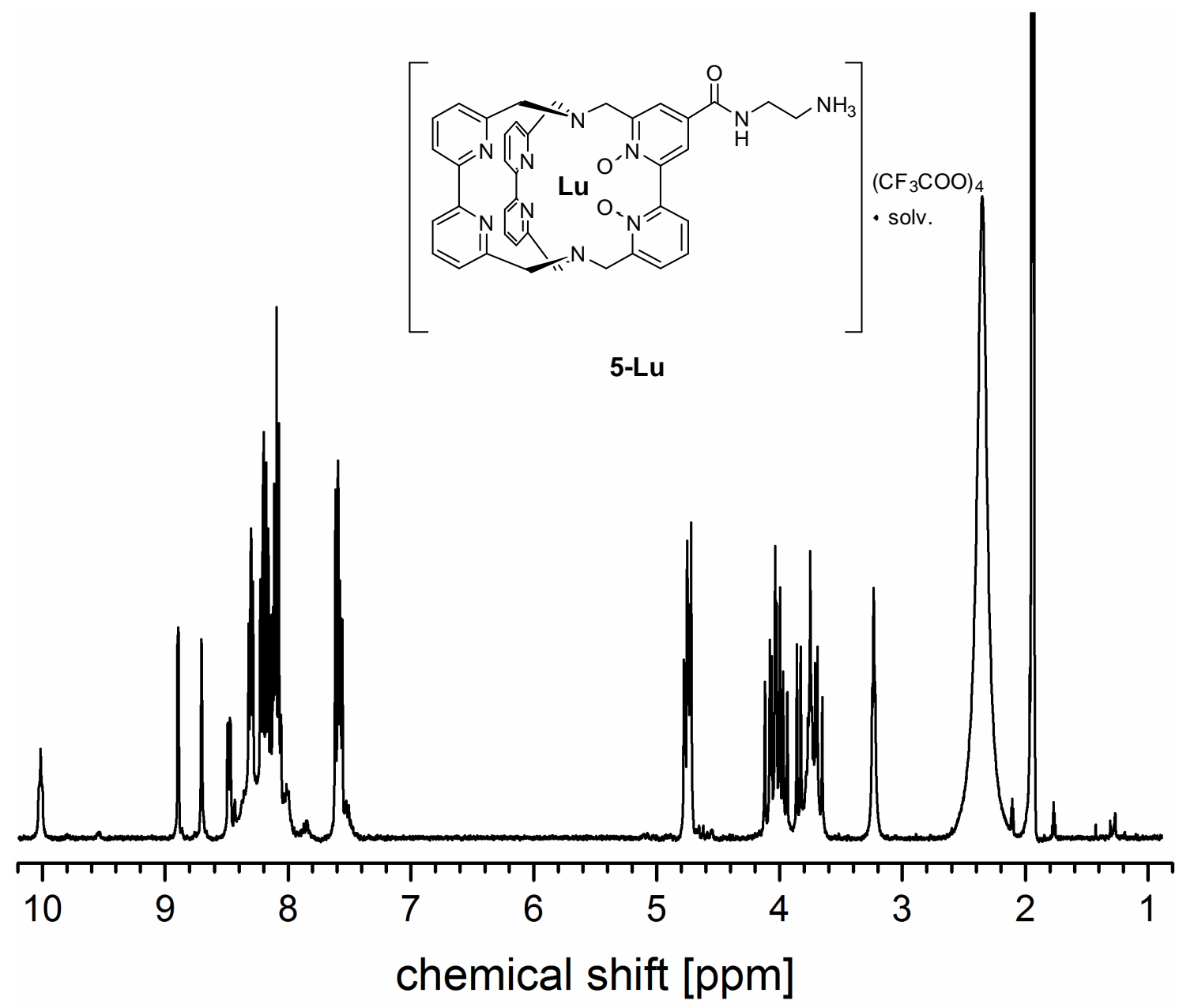

Figure S12. ${ }^{1} \mathrm{H}$ NMR $\left(400 \mathrm{MHz}, \mathrm{CD}_{3} \mathrm{CN}\right)$ of 5-Lu. 


\section{HPLC traces of 5-Ln}

For the detailed HPLC conditions see the main article.

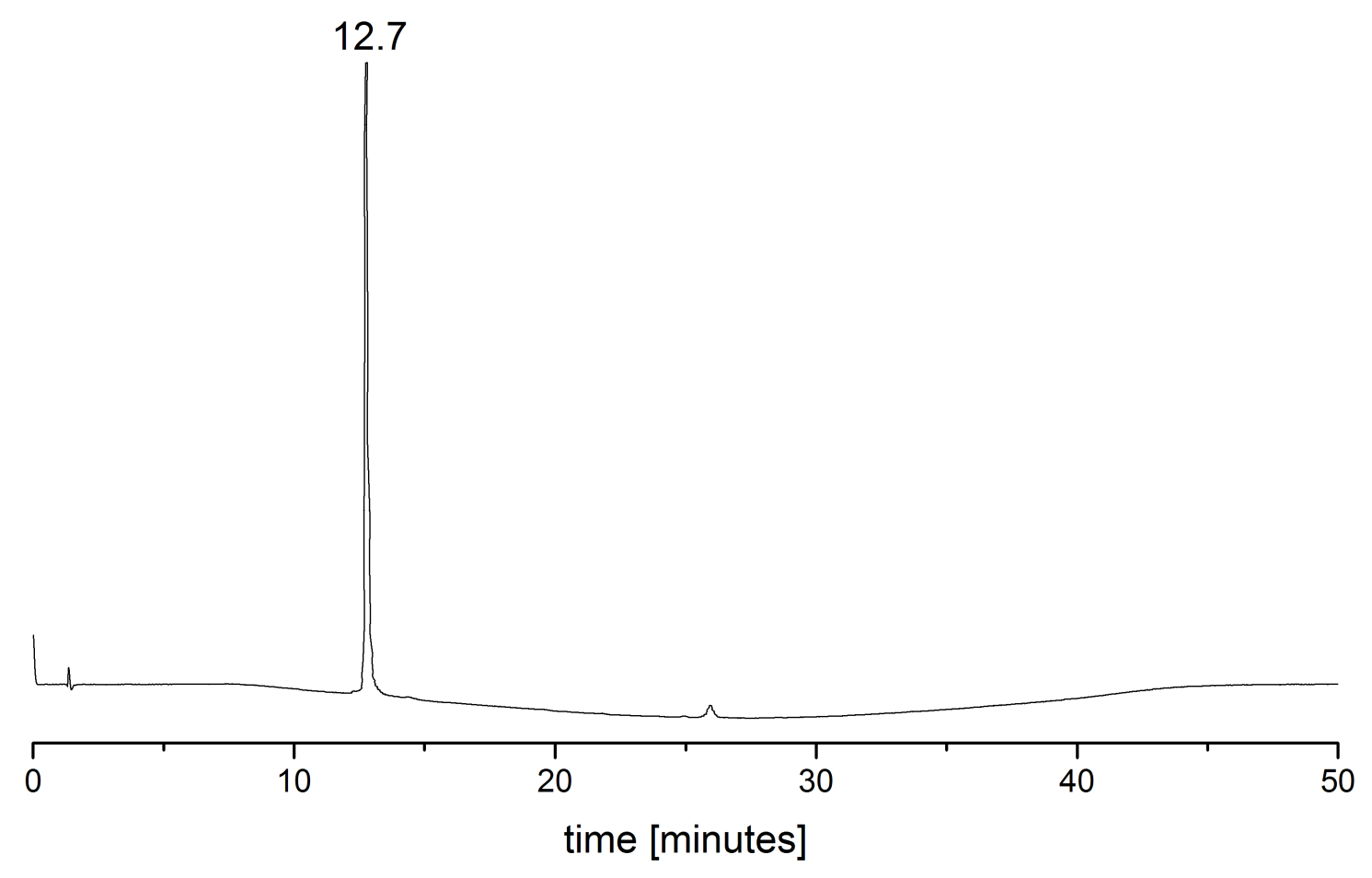

Figure S13. Analytical HPLC trace of 5-Yb (after preparative HPLC purification).

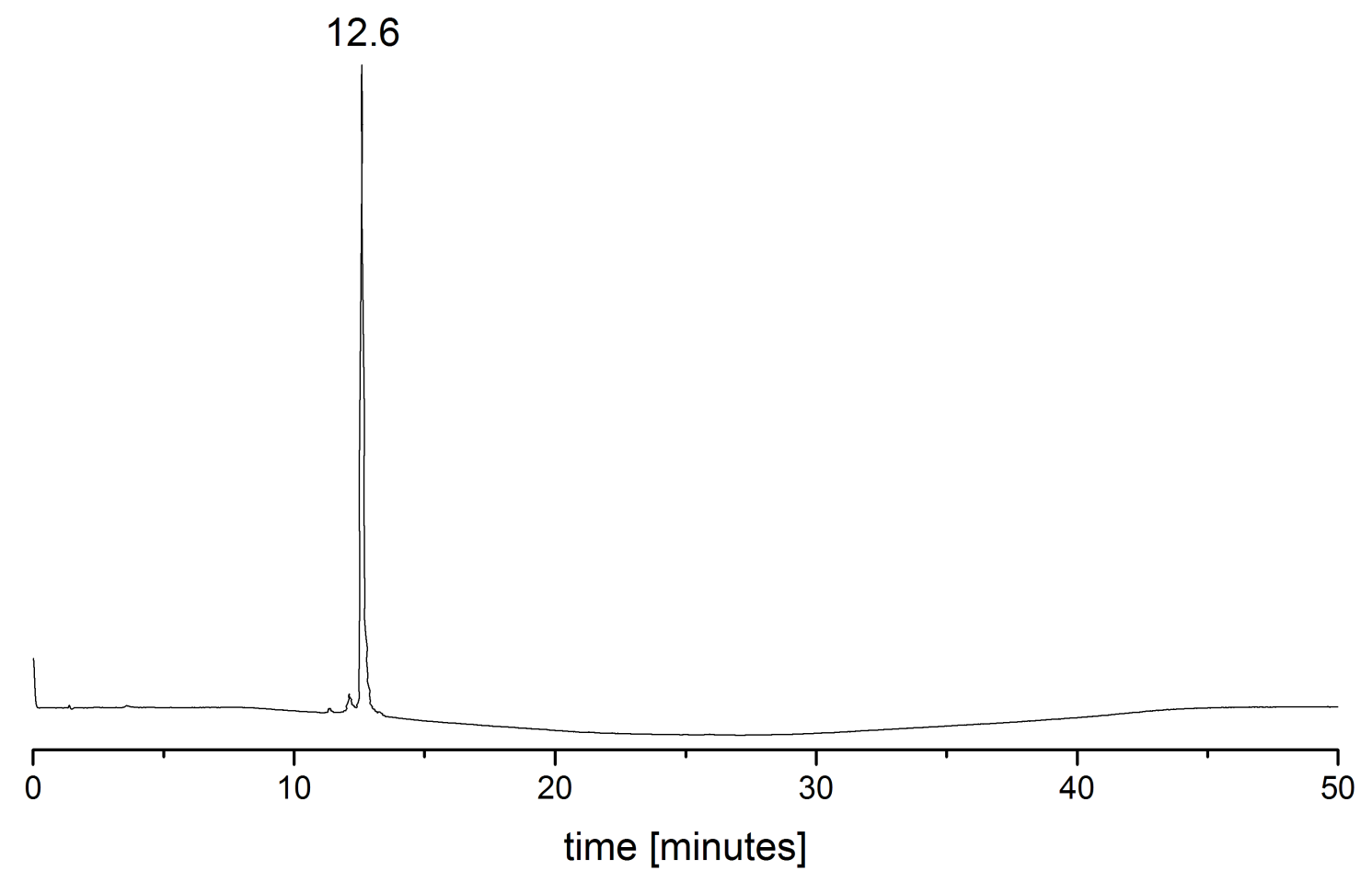

Figure S14. Analytical HPLC trace of 5-Lu (after preparative HPLC purification). 


\section{DFT Calculations}

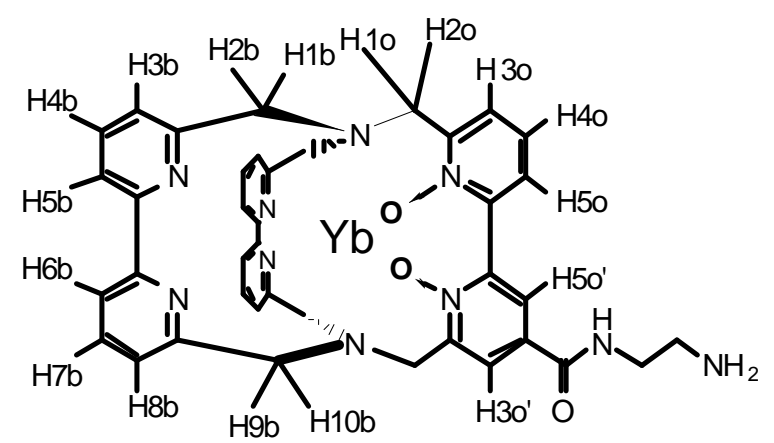

Figure S15. Numbering scheme for the hydrogen atoms in 5-Yb.

Cartesian coordinates for the calculated structure of $5-\mathrm{Yb}$ in acetonitrile:

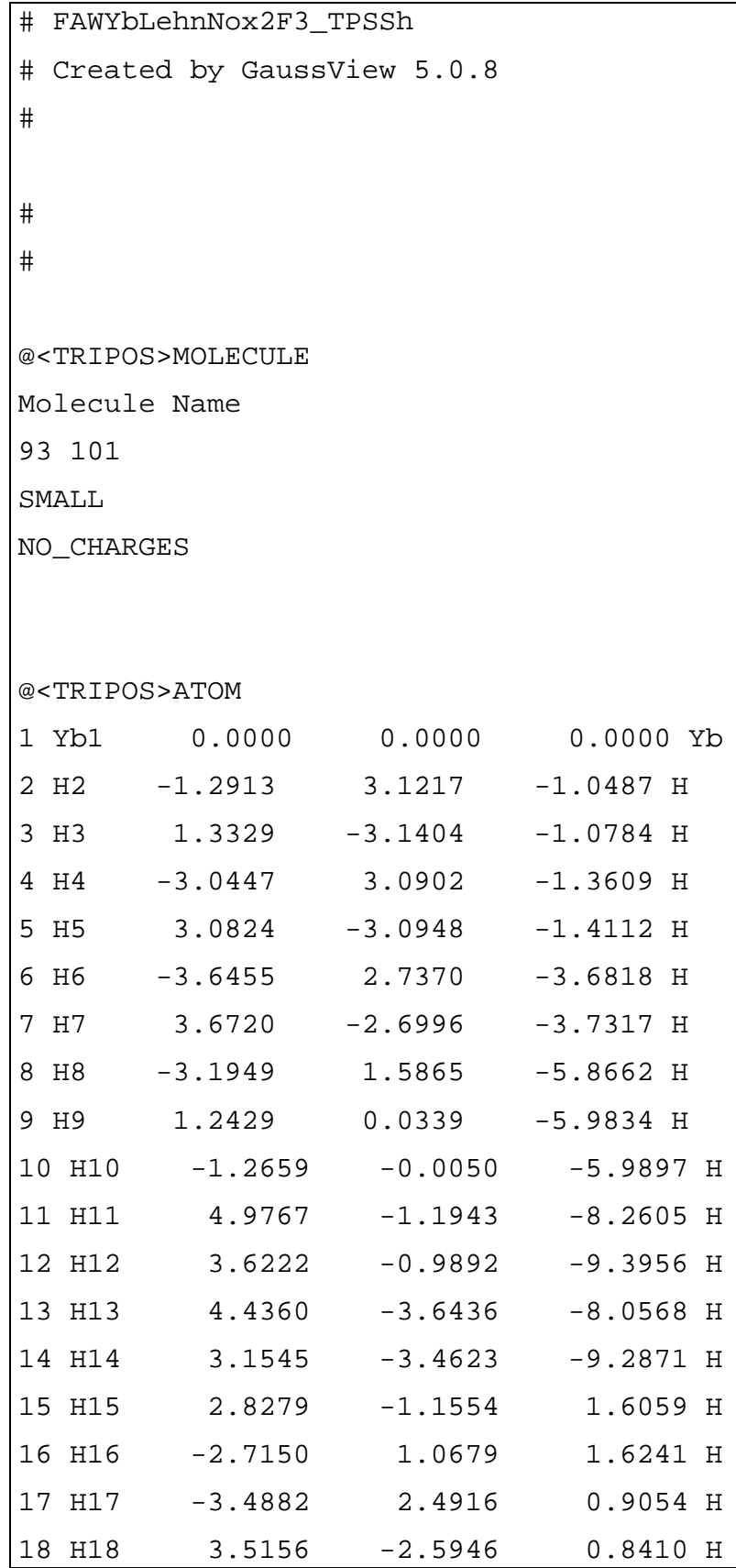




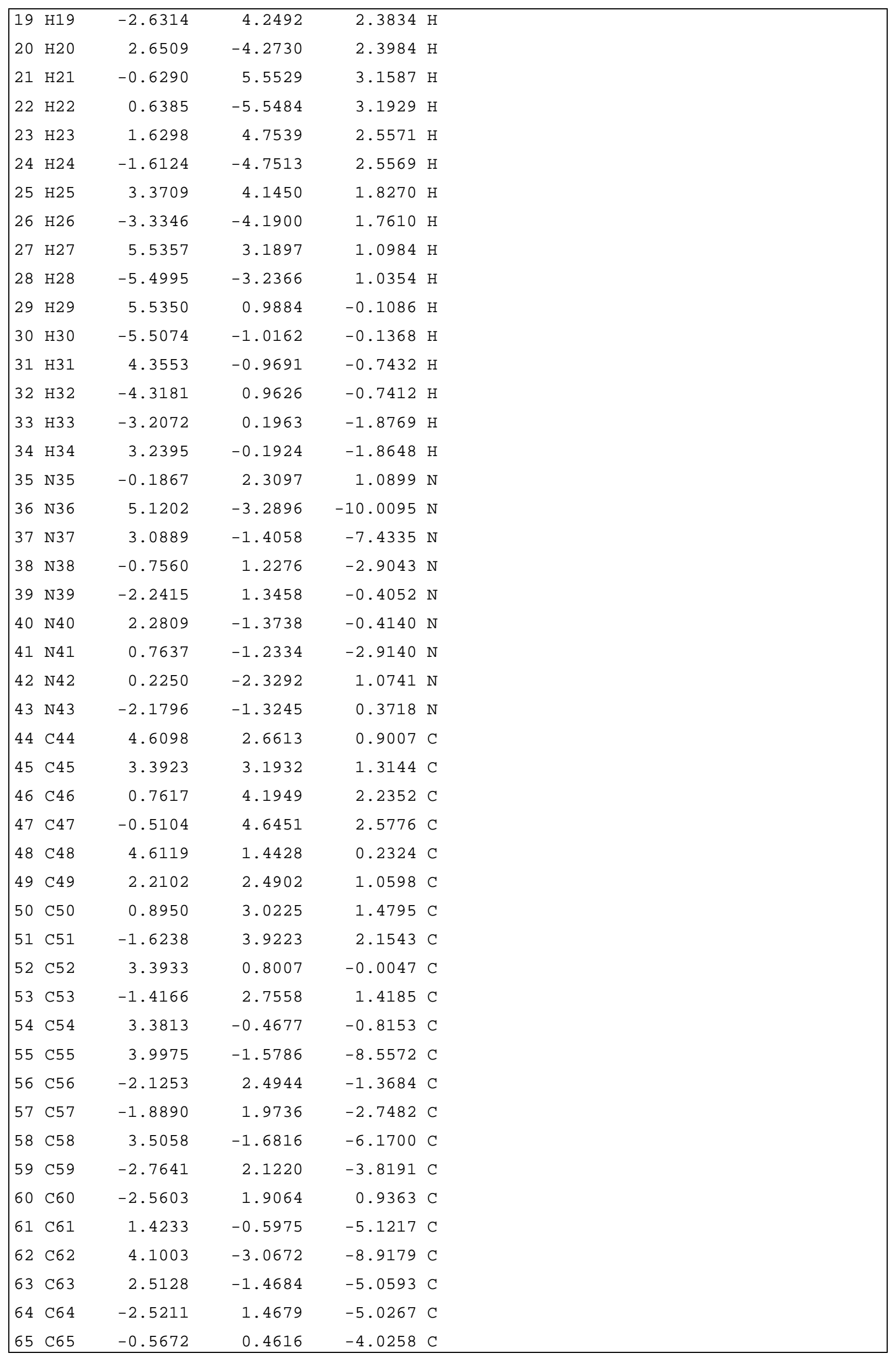




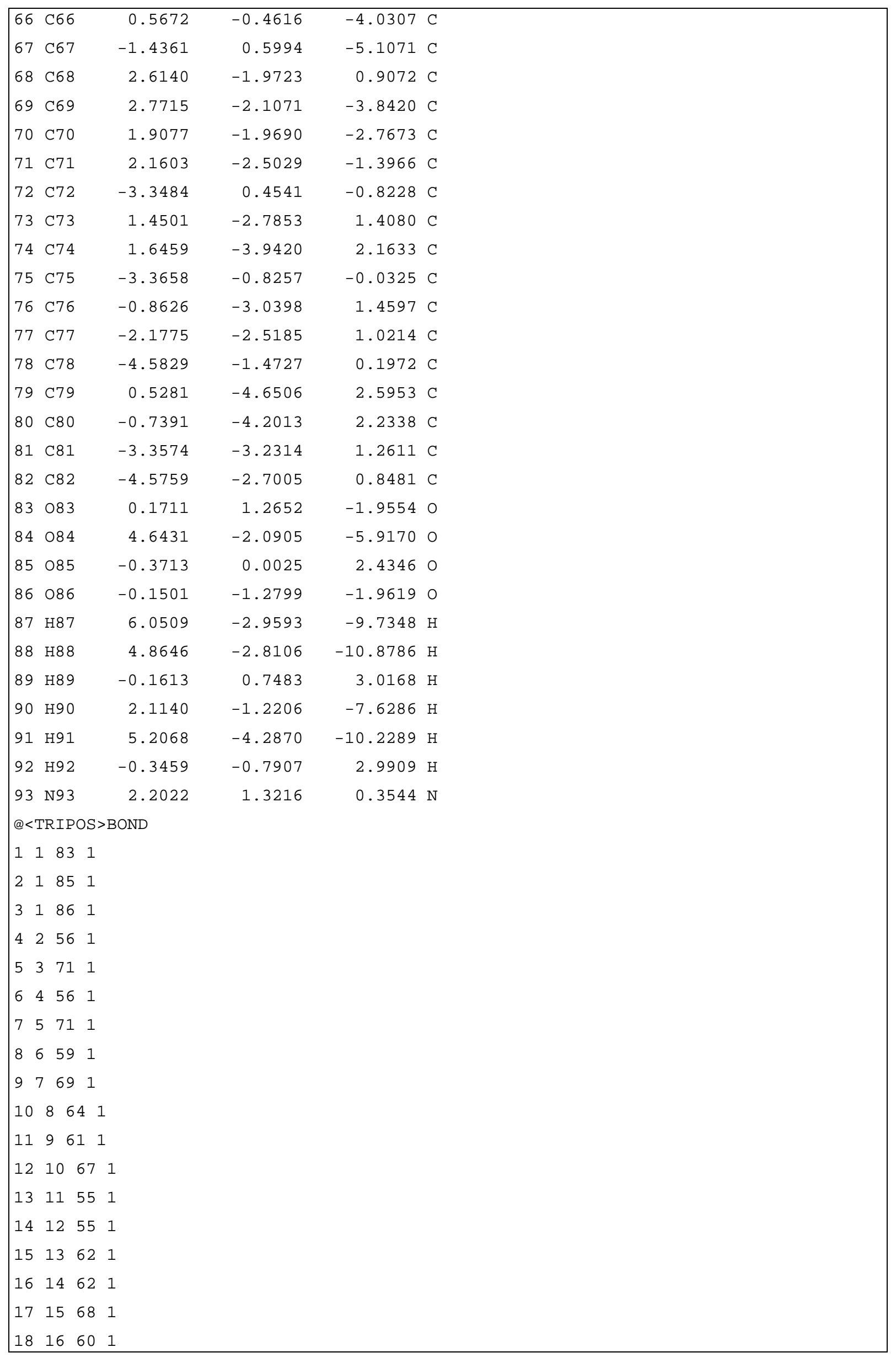




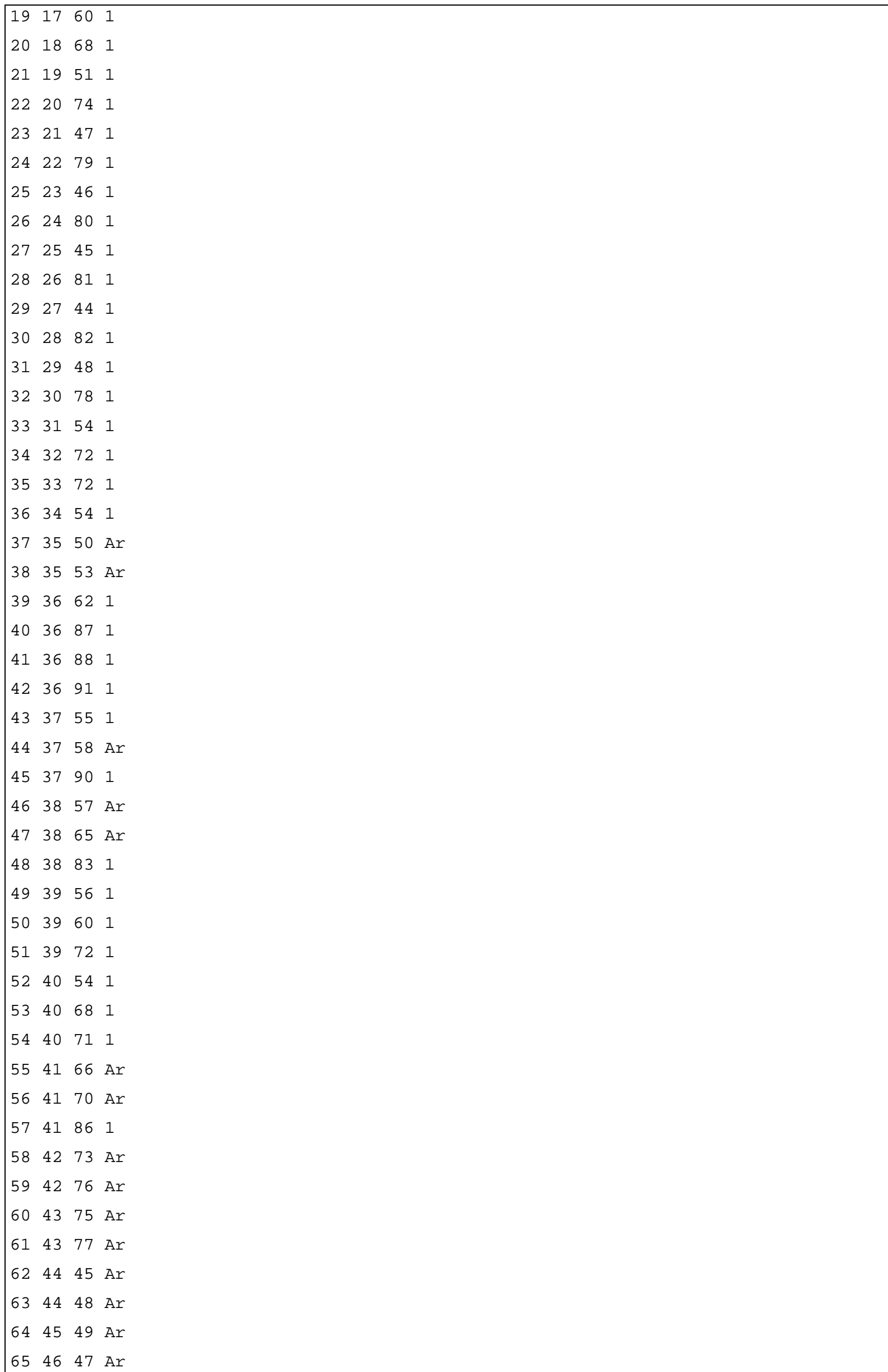




$$
\begin{aligned}
& \begin{array}{llll}
66 & 4650 & \text { Ar }
\end{array} \\
& 67 \quad 4751 \text { Ar } \\
& 68 \quad 4852 \text { Ar } \\
& \begin{array}{llll}
69 & 49 & 50 & 1
\end{array} \\
& 70 \quad 4993 \text { Ar } \\
& 715153 \mathrm{Ar} \\
& \begin{array}{llll}
72 & 52 & 54 & 1
\end{array} \\
& 73 \quad 5293 \mathrm{Ar} \\
& \begin{array}{llll}
74 & 53 & 60 & 1
\end{array} \\
& \begin{array}{llll}
75 & 55 & 62 & 1
\end{array} \\
& \begin{array}{llll}
76 & 56 & 57 & 1
\end{array} \\
& \begin{array}{llll}
77 & 57 \quad 59 & \text { Ar }
\end{array} \\
& \begin{array}{llll}
78 & 58 & 63 & 1
\end{array} \\
& \begin{array}{llll}
79 & 58 & 84 & 2
\end{array} \\
& 805964 \text { Ar } \\
& 816163 \mathrm{Ar} \\
& 8261 \quad 66 \text { Ar } \\
& 8363 \quad 69 \text { Ar } \\
& 84 \quad 64 \quad 67 \text { Ar } \\
& \begin{array}{llll}
85 & 65 & 66 & 1
\end{array} \\
& 86 \quad 65 \quad 67 \mathrm{Ar} \\
& \begin{array}{llll}
87 & 68 & 73 & 1
\end{array} \\
& \begin{array}{llll}
88 & 69 & 70 & 2
\end{array} \\
& \begin{array}{llll}
89 & 70 & 71 & 1
\end{array} \\
& \begin{array}{llll}
90 & 72 & 75 & 1
\end{array} \\
& 9173 \quad 74 \text { Ar } \\
& 927479 \text { Ar } \\
& 93 \quad 75 \quad 78 \mathrm{Ar} \\
& \begin{array}{llll}
94 & 76 & 77 & 1
\end{array} \\
& 9576 \quad 80 \mathrm{Ar} \\
& 967781 \text { Ar } \\
& 97 \quad 78 \quad 82 \text { Ar } \\
& 987980 \mathrm{Ar} \\
& 9981 \quad 82 \text { Ar } \\
& \begin{array}{lllll}
100 & 85 & 89 & 1
\end{array} \\
& \begin{array}{llll}
101 & 85 & 92 & 1
\end{array}
\end{aligned}
$$

